\title{
Murs de soutènement ancrés : modélisation de la rupture le long de la surface de glissement profonde
}

L. BELABED

Institut de Génie civil Centre universitaire

de Guelma, BP 401 24000 Guelma, Algérie

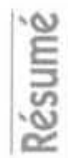

\begin{abstract}
La vérification de la stabilité d'ensemble (mur-solancrage) des murs de soutènement ancrés et la détermination des longueurs d'ancrages nécessaires s'effectuent en étudiant généralement la rupture le long de la surface de glissement profonde. Le modèle mécanique approprié à ce mode de rupture est sujet à controverse. Dans cet article on propose un modèle mécanique de calcul pour la rupture le long de la surface de glissement profonde basé sur la méthode cinématique des solides rigides (analyse limite). Une comparaison de ce modèle proposé avec le modèle conventionnel a été menée selon le concept statistico-probabiliste de sécurité. La fiabilité du système est exprimée au moyen de l'indice de fiabilité. Enfin, des recommandations sont données pour la modélisation de la rupture le long de la surface de glissement profonde.
\end{abstract}

Mots-clés : murs de soutènement, ancrages, analyse limite, stabilité, rupture.

The assessment of the overall stability (wall-ground-anchor) and the determination of anchor lengths in anchored retaining walls are generally carried out based on the investigation of the failure along the deep slip surface. The appropriate mechanical model related to this mode of failure is controversial. In this paper, a mechanical model based on the kinematic theory of rigid bodies is proposed for the failure along the deep slip surface. A comparison of the model proposed with the conventional model is carried out in relation with the statistic-probabilistic safety concept. The system safety is estimated by the reliability index. Finally, recommendations are given for a modelling of the failure along the deep slip surface.

Key words : retaining walls, anchors, limit analysis, stability, failure. 


\section{LISTE DES SYMBOLES}

poids spécifique du sol.

frottement actif mur-sol.

frottement passif mur-sol.

inclinaison de l'ancrage avec l'horizontale.

inclinaison de la surface de glissement avec I'horizontale.

angle de frottement interne.

force d'ancrage.

force de cohésion.

force de pression active du sol.

force de pression passive du sol.

poids propre du solide de glissement $i$.

longueur d'ancrage.

charge proportionnelle au solide de glissement i.

résultante des forces horizontales et verticales.

interaction des solides rigides 1 et 2 .

cohésion.

hauteur du mur.

surcharge illimitée.

surcharge limitée.

fiche du mur dans le sol.

\section{Introduction}

La construction de fouilles profondes a gagné de plus en plus d'importance dans les dernières décennies, en particulier dans les centres des villes (construction de parkings souterrains, tunnels du métro souterrain, etc.). Les murs ancrés assurent vis-à-vis des murs butonnés une bonne liberté de mouvement à l'intérieur de la fouille. Les ancrages précontraints injectés sont fréquemment utilisés de nos jours dans la pratique.

L'expérience pratique et des études théoriques [2, 8, 14] ont montré que, pour la vérification de la stabilité d'ensemble (mur-sol-ancrage) des murs de soutènement ancrés et la détermination des longueurs d'ancrages nécessaires, on doit étudier généralement la rupture le long de la surface de glissement profonde. La vérification de la stabilité le long de la surface de glissement profonde a été traitée dans la littérature spécialisée avec différents procédés [1, 2, 9, 13]. Kranz [13] a proposé, pour la première fois, un modèle mécanique de rupture le long de la surface de glissement profonde (ab) pour les murs de soutènement ancrés à un mur ou à une plaque d'ancrage (Fig. 1a). Plus tard, ce modèle a été repris pour les ancrages précontraints injectés en plaçant un mur d'ancrage vertical fictif au point d'intersection de la surface de glissement profonde (ab) avec l'ancrage injecté (Fig. 1b), afin de permettre l'application de la méthode de Kranz également aux ancrages injectés. Cette approche n'est pas toujours précise et pourrait conduire à une sous-estimation de la sécurité du système. Ce problème fait l'objet du présent article.

Des essais sur modèles [8] et des investigations théoriques des murs de soutènement ancrés avec des ancrages précontraints injectés [2, 3] ont montré que les mécanismes de rupture basés sur la théorie de la cinématique des solides rigides (analyse limite) reflètent mieux la rupture réelle. Selon cette dernière théorie, un mécanisme de rupture est constitué de plusieurs solides rigides de glissement et surfaces de glissement planes de part et d'autre du mur (Fig. 1c). L'application de la méthode cinématique des solides rigides à la modélisation des mécanismes de rupture implique une translation du mur. Pour plus de détails sur cette théorie voir [2, 7, 9].

Des comparaisons de calculs [2] ont montré que les deux solides de rupture devant le pied du mur (Fig. 1c) peuvent être remplacés, avec une exactitude suffisante, par la butée E de Gudehus/Gross [11].

L'analyse de la sécurité (fiabilité) du système est basée sur le concept statistico-probabiliste de sécurité selon Grusibau [10] « Principes fixant les exigences en matière de sécurité des constructions ) et DIN 1054.100 [6] édités par l'Institut allemand de normalisation (DIN), ainsi que Eurocode 7 [5]. En se basant sur les résultats des travaux réalisés au département de mécanique des sols à l'université de Weimar [14], les données statistiques des variables aléatoires et les facteurs partiels de sécurité sont regroupés dans le tableau $\mathrm{l}$.

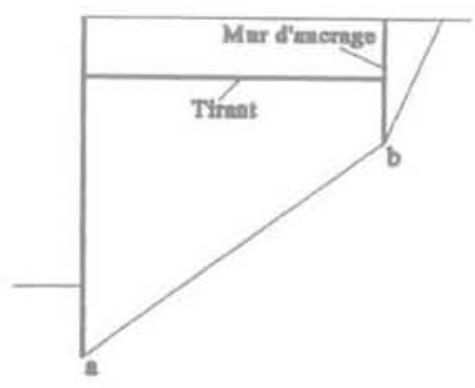

(a)

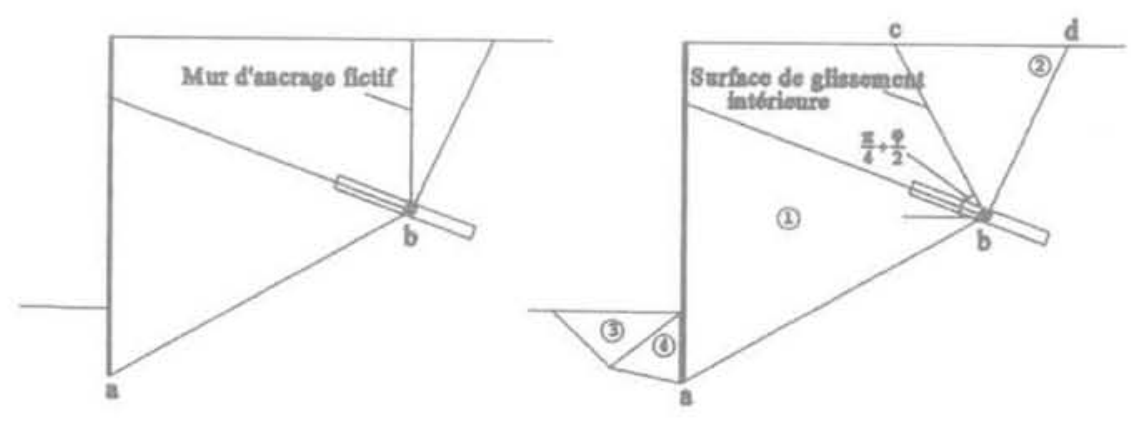

(b)

(c)

FG.1 Rupture le long de la surface de glissement profonde. a) Modèle de rupture d'après Kranz [13] ; b) Modèle simplifié pour ancrage injecté ; c) Modèle cinématique pour ancrage injecté.

Fallure in the deep slip surface.

a) Failure model after Kranz [13] ; b) Simplified model for grouted anchor; c) Kinematical model for grouted anchor. 
La sécurité du système est exprimée au moyen de l'indice de fiabilité $\beta$ calculé avec la théorie de fiabilité de premier ordre. Les équations d'états-limites (4, 5, 6 et 7 ), correspondant à chaque modèle mécanique, sont indispensables pour toute étude déterministe (longueurs d'ancrages) et probabiliste (indices de fiabilité). La formulation des équations d'états limites se fait en étudiant l'équilibre des forces horizontales et verticales de chaque solide séparément. Pour plus de détails voir [4].

\section{Comparaison des modèles mécaniques}

Dans cette étude on fait une comparaison entre le modèle cinématique basé sur la méthode cinématique des solides rigides (Fig. 1c) et le modèle simplifié (Fig. 1b). Ce dernier est caractérisé par un mur d'ancrage vertical fictif placé au point d'intersection de la surface de glissement profonde (ab) avec l'ancrage au lieu d'une surface de glissement intérieure inclinée. C'est la seule différence entre les deux modèles précités.

\section{Influence des forces d'ancrages}

\section{1}

Cas 1 : aucune force d'ancrage n'agit sur le solide situé à l'arrière

Pour les modèles cinématiques A et B (Fig. 2), aucune force d'ancrage n'agit sur le solide actif numéro 2, situé à l'arrière. Des analyses comparatives [2] ont montré que la résuitante $Q_{12}$ (interaction des solides 1 et 2) de ce solide peut être remplacée par la force de pression active du sol $\mathrm{E}_{\text {. }}$ (voir équations 4 et 5 ). Pour les modèles simplifiés A'et 'B', non présentés dans ce travail, une force de pression active du sol $E_{u^{\prime}}$, est appliquée sur le mur d'ancrage vertical fictif.

Pour formuler l'équation d'état limite correspondant au modèle A, représenté sur la figure 2, on doit étudier l'équilibre des solides rigides (1) et (2) séparément. Le solide (2) a été remplacé par la force de pression active du sol $E_{\text {a: }}$

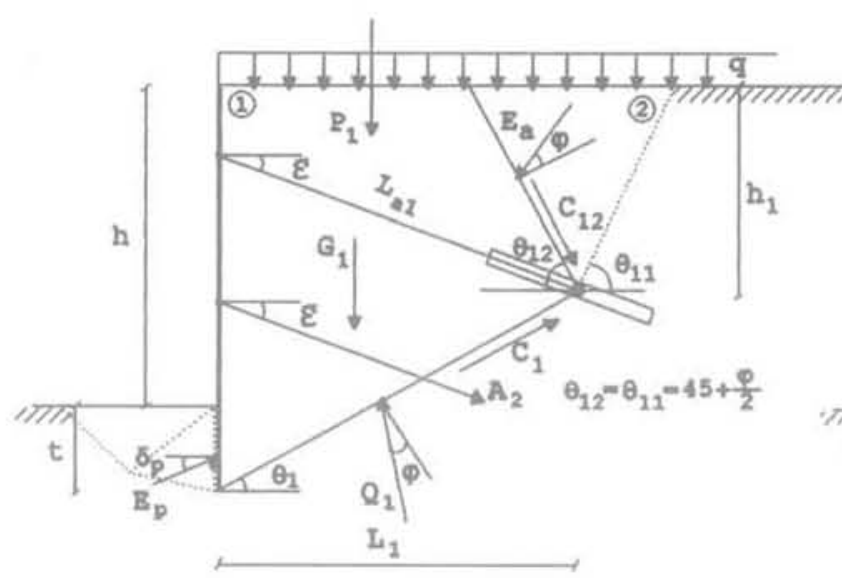

Modele A

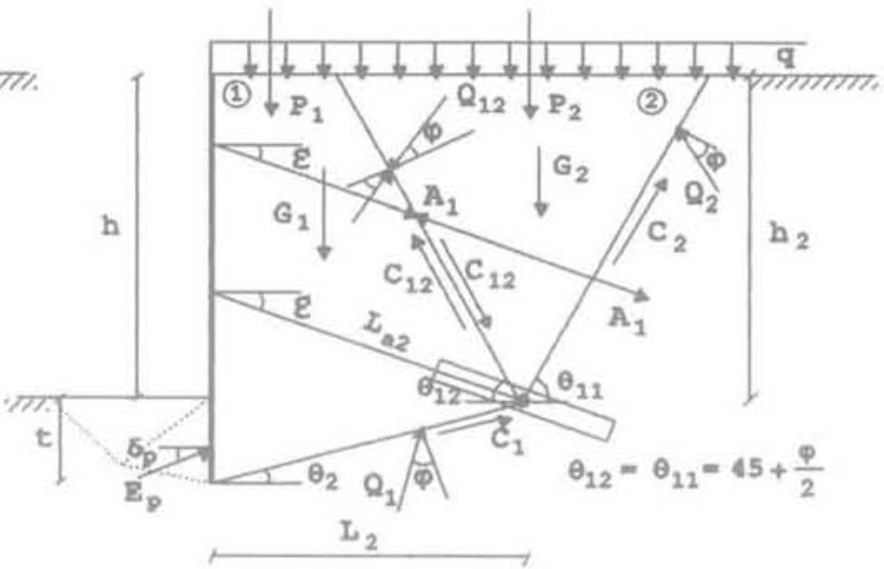

Modele B

FIG 2 Modèles de rupture cinématiques pour un mur à double ancrage (cas 1). Kinematical failure models for a double-propped retaining wall (case 1),

TABLEAU1 Données statistiques des variables aléatoires et facteurs partiels de sécurité. Statistical data of the basic variables and partial reliability factors.

\begin{tabular}{l|c|c|l|c}
\hline Variable aleatoire & Valeur moyerne & Coelficient de variation & Distribution & Facteur partiel de sécurité \\
\hline Frottement interne $\varphi\left({ }^{\circ}\right)$ & $20-40$ & $7,5 \%$ & Log-normale & 1,25 \\
\hline Cohésion $c(\mathrm{kPa})$ & $35-0$ & $25 \%$ & Log-normale & 1,6 \\
\hline Surcharge $q(\mathrm{kPa})$ & 10 & $40 \%$ & Extréme (type $\mathrm{n})$ & 1,3 \\
\hline Poids spécifique $\gamma\left(\mathrm{kN} / \mathrm{m}^{7}\right)$ & 20 & $5 \%$ & Normale & 1,0 \\
\hline
\end{tabular}


L'équilibre des forces horizontales donne:

$E_{a} \cos \left(\frac{\pi}{4}+\frac{\varphi}{2}\right)-C_{12} \cos \theta_{12}-C_{1} \cos \theta_{1}-E_{p} \cos \delta_{p}$

$-A_{2} \cos \varepsilon+Q_{1} \sin \left(\theta_{1}-\varphi\right)=0$

L'équilibre des forces verticales donne:

$G_{1}+P_{1}+E_{\mathrm{a}} \sin \left(\frac{\pi}{4}+\frac{\varphi}{2}\right)+C_{12} \sin \theta_{12}-C_{1} \sin \theta_{1}$

$-E_{p} \sin \delta_{p}+A_{2} \sin \varepsilon-Q_{1} \cos \left(\theta_{1}-\varphi\right)=0$

De l'équation (1) on tire :

$$
\begin{array}{r}
-E_{2} \cos \left(\frac{\pi}{4}+\frac{\varphi}{2}\right)+C_{12} \cos \theta_{12}+C_{1} \cos \theta_{1} \\
Q_{1}=\frac{+E_{p} \cos \delta_{p}+A_{2} \cos \varepsilon}{\sin \left(\theta_{1}-\varphi\right)}
\end{array}
$$

En substituant l'équation (3) dans l'équation (2) et avec :

$$
\theta_{12}=\frac{\pi}{4}+\frac{\varphi}{2}
$$

on obtient après transformations algébriques l'équation d'état-limite correspondant à la rupture au niveau de l'ancrage supérieur d'un mur de soutènement à double ancrage, modèle $\mathrm{A}$ (Fig. 2) :

$$
\begin{aligned}
& E_{p} \cos \left(\delta_{p}-\theta_{1}+\varphi\right)+C_{1} \cos \varphi \\
& +C_{12} \cos \left(\frac{\pi}{4}-\frac{\varphi}{2}+\theta_{1}\right)+A_{2} \cos \left(\theta_{1}-\varphi+\varepsilon\right) \\
& -\left\{\left(G_{1}+P_{1}\right) \sin \left(\theta_{1}-\varphi\right)+E_{2} \cos \left(\frac{\pi}{4}+\frac{3 \varphi}{2}-\theta_{1}\right)\right\}=0
\end{aligned}
$$

On procède de la mème manière pour développer les équations d'états limites ci-dessous correspondants aux différents modèles mécaniques.

L'équation d'état-limite correspondant à la rupture au niveau de l'ancrage inférieur d'un mur de soutènement à double ancrage, modèle B (Fig. 2) est:

$$
\begin{aligned}
& E_{p} \cos \left(\delta_{p}-\theta_{2}+\varphi\right)+C_{1} \cos \varphi \\
& +C_{12} \cos \left(\frac{\pi}{4}+\theta_{2}-\frac{\varphi}{2}\right)+A_{1} \cos \left(\theta_{2}-\varphi+\varepsilon\right) \\
& -\left\{\left(G_{1}+P_{1}\right) \sin \left(\theta_{2}-\varphi\right)+E_{2} \cos \left(\frac{\pi}{4}+\frac{3 \varphi}{2}-\theta_{2}\right)\right\}=0
\end{aligned}
$$

Les résultats des études paramétriques illustrés sur la figure 3 montrent que les deux modèles cinématique et simplifié donnent non seulement les mêmes longueurs d'ancrages, mais encore les mêmes indices de sécurité. Dans ce cas, le modèle simplifié est une bonne approche pour le modèle cinématique. L'introduction d'un mur d'ancrage vertical fictif, au lieu d'une surface de glissement intérieure inclinée, est donc une bonne approximation et sert à simplifier l'étude du problème.

\section{Cas 1}

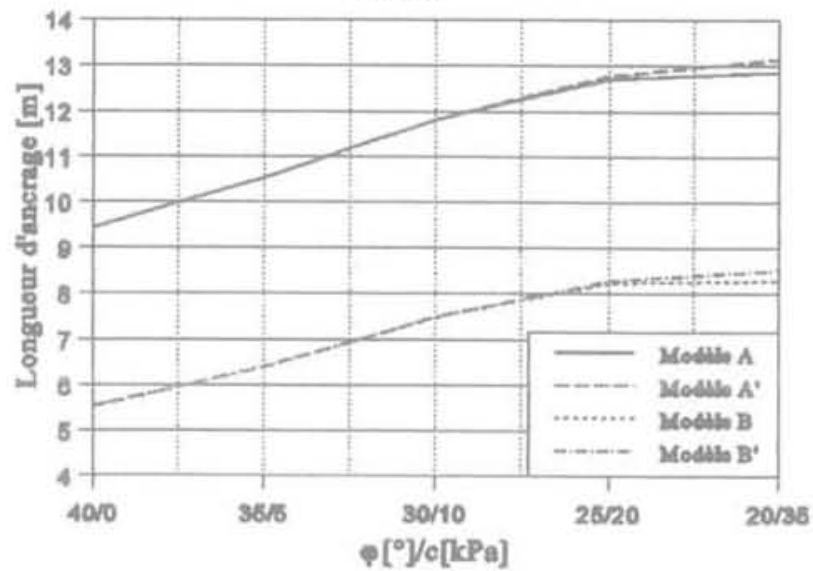

Cas 1

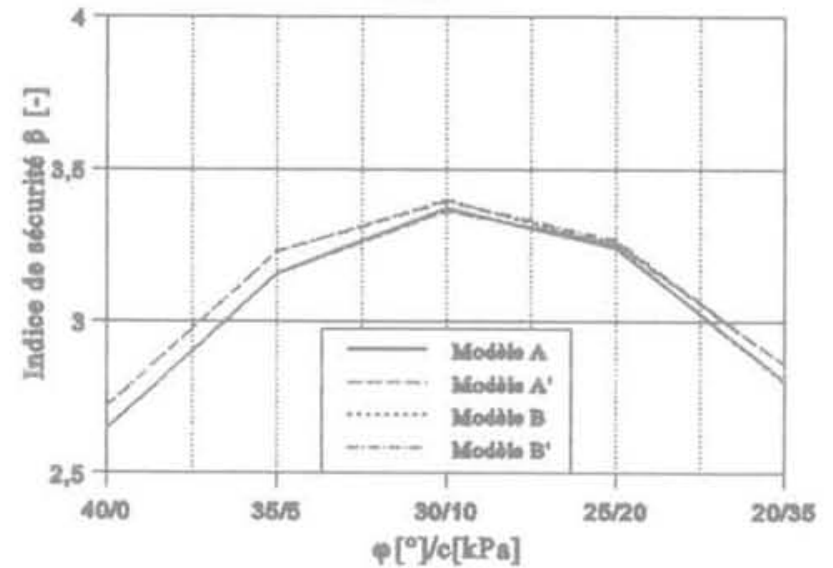

FG. 3 Comparaison des modèles de rupture cinématiques et simplifiés (cas 1).

Comparison of kinematical and simplified failure models (case 1).

\section{1 .2}

\section{Cas 2 : Des forces d'ancrages agissant sur le solide situé à l'arrière}

Pour les modèles cinématiques $C$ et D (Fig. 4), les forces d'ancrages $\mathrm{A}_{1}$ ou $\mathrm{A}_{\text {, }}$ et $\mathrm{A}_{2}$ agissent sur le solide actif numéro 2, situé à l'arrière, parce que les ancrages correspondants sont coupés une seule fois par la surface de glissement intérieure. Des comparaisons de calculs [2] ont montré que la résultante $\left(Q_{12}\right)$ agissant sur la surface de glissement intérieure est beaucoup plus grande que la force de pression active du sol $\mathrm{E}_{\mathrm{e}}$. C'est pourquoi, contrairement au premier cas, on ne peut pas remplacer le solide (2) par la force de pression active du sol sinon un sous-dimensionnement est à craindre. Cela est dû principalement aux forces d'ancrages coupés qui empêchent le sol d'atteindre l'état limite actif.

Pour les modèles simplifiés C' et D' (Fig. 5), I'introduction d'un mur d'ancrage vertical fictif, sur lequel la force de pression active du sol $E_{\text {a }}$ est appliquée, ne reflète pas la réalité puisque l'effet des forces d'ancrages coupés est négligé. 

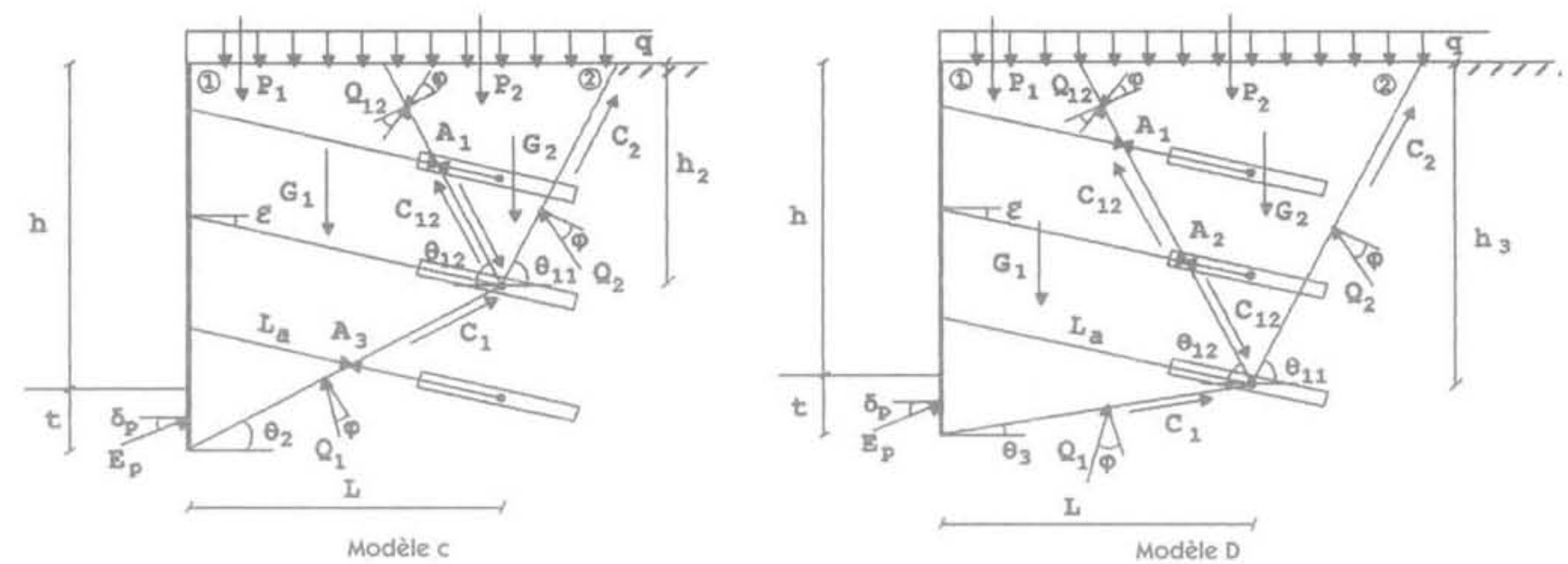

flG.4 Modèles de rupture cinématiques pour un mur à triple ancrage (cas 2). Kinematical failure models for a triple-propped retaining wall (case 2).

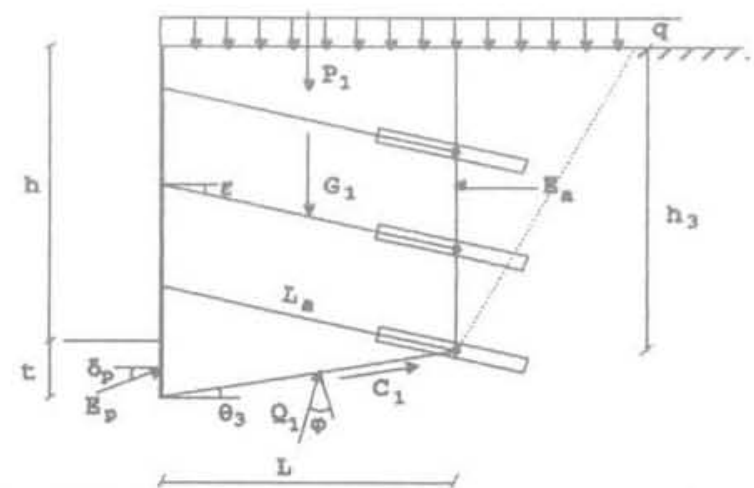

FIG.5. Modèle simplifié : rupture au niveau de l'ancrage inférieur d'un mur à triple ancrage (modèle D').

Simplified model : failure of the bottom anchor of a triple-propped retaining wall (model D').

L'équation d'état limite correspondant à la rupture au niveau de l'ancrage du milieu d'un mur de soutènement à triple ancrage, modèle C (Fig. 4) est :

$E_{p} \cos \left(\delta_{p}-\theta_{2}+\varphi\right)+C_{1} \cos \varphi+C_{12} \cos \left(\theta_{12}-\varphi+\theta_{2}\right)+$ $C_{2} m_{2} \cos \varphi+\left(A_{1}+A_{3}\right) \cos \left(\theta_{2}-\varphi+\varepsilon\right)-l\left(G_{1}+P_{1}\right) \sin \left(\theta_{2}-\varphi\right)+$ $\left[\left(G_{2}+P_{2}\right) \sin \left(\theta_{14}-\varphi\right)+A_{1} \cos \left(\theta_{13}-\varphi+\varepsilon\right)\right] m_{2} \mid=0$

avec:

$m_{2}=\frac{\sin \left(\theta_{12}-2 \varphi+\theta_{2}\right)}{\sin \left(\theta_{12}+\theta_{11}-2 \varphi\right)}$

L'équation d'état limite correspondant à la rupture au niveau de l'ancrage inférieur d'un mur de soutènement à triple ancrage, modèle D (Fig. 4) est :

$E_{p} \cos \left(\delta_{p}-\theta_{3}+\varphi\right)+C_{1} \cos \varphi+C_{12} \cos \left(\theta_{12}-\varphi+\theta_{3}\right)+C_{2} m_{3} \cos \varphi$ $+\left(A_{1}+A_{2}\right) \cos \left(\theta_{3}-\varphi+\varepsilon\right)-\left[\left(G_{1}+P_{1}\right) \sin \left(\theta_{3}-\varphi\right)+\left[\left(G_{2}+\right.\right.\right.$ $\left.\left.P_{2}\right) \sin \left(\theta_{11}-\varphi\right)+\left(A_{1}+A_{2}\right) \cos \left(\theta_{11}-\varphi+\varepsilon\right)\right] m_{3} \mid=0$

avec :

$m_{3}=\frac{\sin \left(\theta_{12}-2 \varphi+\theta_{3}\right)}{\sin \left(\theta_{12}+\theta_{11}-2 \varphi\right)}$

Les résultats des calculs sont représentés sur la figure 6. Dans le cas de la rupture au niveau de l'ancrage du milieu, le modèle simplifié C' donne des ancrages plus longs que le modèle cinématique C. La différence maximale est à peu près $7 \%$. La différence est clairement plus grande (environ $20 \%$ ) dans le cas de la rupture au niveau de l'ancrage inférieur (modèles D et D', voir tableaux II et III). Tout de même, les deux modèles présentent les mêmes indices de fiabilité. Dans ce cas, il est déconseillé d'étudier le modèle simplifié à la place du modèle cinématique puisque le modèle simplifié sous-estime ici la sécurité. Une modélisation correcte des mécanismes de rupture est garantie seulement avec le modèle cinématique parce qu'avec ce dernier la sécurité du système n'est pas sous-estimée et correctement calculée.

De plus, un dimensionnement économique des longueurs d'ancrages (jusqu'à environ 2,00 m plus courtes) est possible si on analyse les mécanismes de rupture avec la méthode cinématique [3] (voir Fig. 6). Du tableau II on constate que le modèle cinématique donne des ancrages plus courts que le modèle simplifié. Cette différence est d'autant plus importante que la cohésion est plus grande. Alors que leurs indices de fiabilité $\beta$ sont égaux (tableau III). Enfin, on a pu souligner que l'influence des forces d'ancrages sur la stabilité des murs de soutènement à ancrage multiple est importante.

Selon les recommandations françaises [12], le modèle simplifié peut être également étudié dans le cas d'ancrages multiples, mais en tenant compte de l'influence des forces d'ancrages coupés sur la force de pression du sol derrière le mur d'ancrage fictif. Reste alors la question sur la manière de déterminer cette force.

\section{2}

\section{Influence d'une surcharge limitée}

L'étude est faite pour une surcharge uniforme limitée des deux côtés (q') et appliquée directement derrière le solide de rupture actif situé à l'avant (Fig. 7). Elle correspond à des charges résultant, par exemple, des constructions adjacentes. On a fait varier sa valeur et sa largeur (b). On suppose que la surcharge limitée est définie par les mèmes paramètres statistiques que la surcharge illimitée q (tableau 1). Les résultats des calculs sont regroupés dans le tableau IV. 
Cas 2

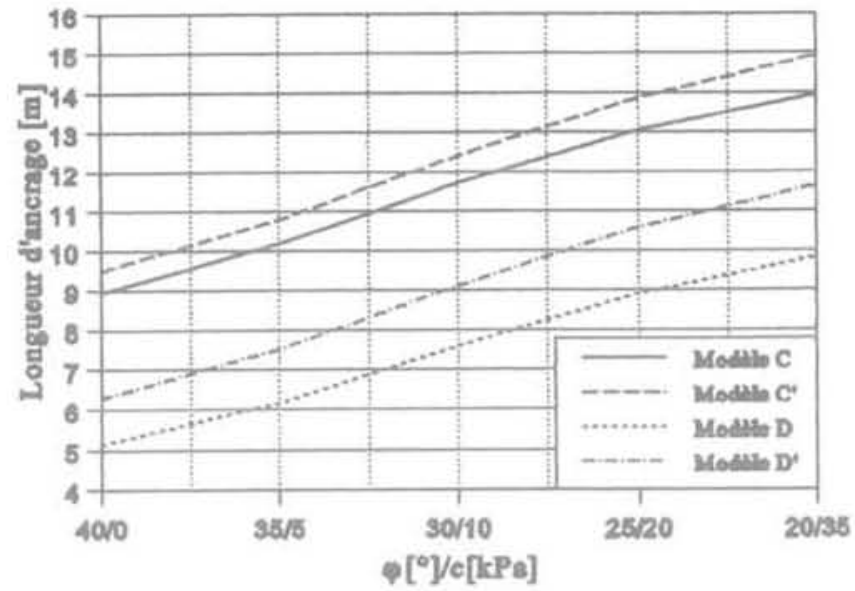

Cas 2

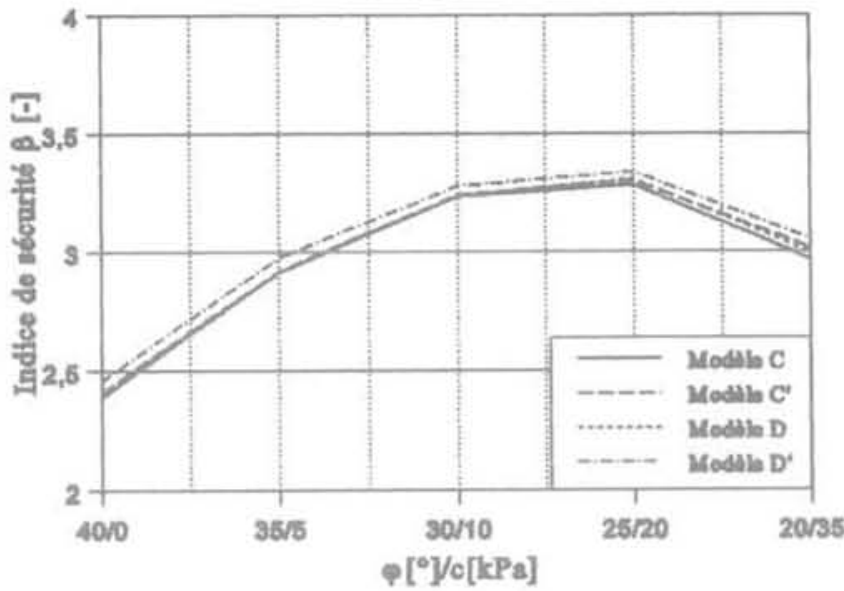

FG.6 Comparaison des modèles de rupture cinématiques et simplifiés (cas 2). Comparison of kinematical and simplified fallure models (case 2).

TAREAUII Comparaison des longueur's d'ancrage (en $\mathrm{m}$ ) des modèles $\mathrm{D}$ et $\mathrm{D}^{\prime}$.

Comparison of the anchor lengths $(m)$ of models $D$ and $D^{\prime}$

\begin{tabular}{l|c|c|c|c|c}
\hline$(\%) / c(k P a)$ & $40 / 0$ & $35 / 5$ & $30 / 10$ & $25 / 20$ & $20 / 35$ \\
\hline $\begin{array}{l}\text { Modèle } \\
\text { cinématique D }\end{array}$ & 5,13 & 6,16 & 7,58 & 8,90 & 9,84 \\
\hline $\begin{array}{l}\text { Modèle } \\
\text { simplifié D }\end{array}$ & 6,28 & 7,49 & 9,11 & 10,57 & 11,65 \\
\hline
\end{tabular}

TABLEAUIII Comparaison des indices de fiabilité (-) des modèles D et D'.

Comparison of reliability indexes of models D and $D^{\prime}$.

\begin{tabular}{l|c|c|c|c|c}
\hline$\varphi(9)$ cikPa) & $40 / 0$ & $35 / 5$ & $30 / 10$ & $25 / 20$ & $20 / 35$ \\
\hline $\begin{array}{l}\text { Modèle } \\
\text { cinématique D }\end{array}$ & 2,40 & 2,91 & 3,24 & 3,31 & 3,00 \\
\hline $\begin{array}{l}\text { Modèle } \\
\text { simplifié D' }\end{array}$ & 2,46 & 2,97 & 3,28 & 3,34 & 3,05 \\
\hline
\end{tabular}

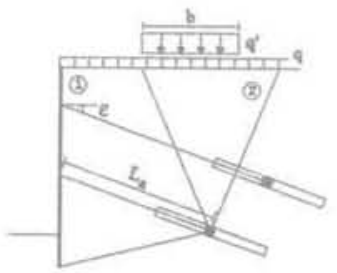

Modde cintanntique B

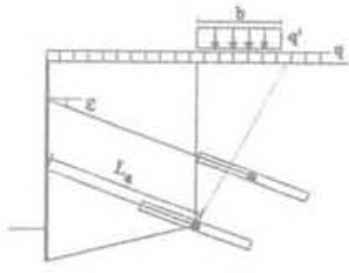

Modele simplifie B'

FIG.7 Influence d'une surcharge limitée. Influence of a both sides limited surcharge.

Des résultats représentés sur le tableau IV on constate que pour une surcharge limitée $q^{\prime}=0$, les deux modèles étudiés, $\mathrm{B}$ et $\mathrm{B}^{\prime}$, donnent les mêmes longueurs d'ancrages et les mêmes indices de fiabilité $\beta$ (voir Fig. 3). Pour $\mathrm{q}^{\prime}=100 \mathrm{kPa}$ et $\mathrm{b}=3 \mathrm{~m}$, le modèle simplifié B' sous-estime la sécurité du système en donnant de plus longs ancrages et légèrement plus petits indices de fiabilité $\beta$ par rapport au modèle cinématique $B$. Cela est attribué à l'hypothèse d'un mur d'ancrage fictif. Les études ont montré que la pression des terres supplémentaire due à q' appliquée sur le mur

TAllEAUN Influence d'une surcharge limitée sur longueurs d'ancrage $L_{a}$ et indices de fiabilité $\beta$. Infiuence of a limited surcharge on anchor lengths $L_{a}$ and reliability indexes $\beta$.

\begin{tabular}{|c|c|c|c|c|c|c|c|c|c|c|}
\hline $\begin{array}{l}\varphi\left({ }^{\circ} / \text { clkPa }\right) \\
\text { Modèle }\end{array}$ & \multicolumn{2}{|c|}{$40 / 0^{\circ}$} & \multicolumn{2}{|c|}{$35 / 5$} & \multicolumn{2}{|c|}{$\begin{array}{c}30 / 10 \\
\mathrm{La}(\mathrm{m}) \beta(-) \\
q^{\prime}=0\end{array}$} & \multicolumn{2}{|c|}{$25 / 20$} & \multicolumn{2}{|c|}{$20 / 35$} \\
\hline B & 5,53 & 2,72 & 6,38 & 3,23 & 7,47 & 3,40 & 8,23 & 3,25 & 8,28 & 2,80 \\
\hline \multirow[t]{2}{*}{$B^{\prime}$} & 5,53 & 2,72 & 6,38 & 3,23 & 7,47 & 3,39 & 8,28 & 3,27 & 8,52 & 2,86 \\
\hline & \multicolumn{10}{|c|}{$\mathrm{q}^{\prime}=30 \mathrm{kPa} \mathrm{b}=1,50 \mathrm{~m}$} \\
\hline B & 5,63 & 2.76 & 6,49 & 3,25 & 7.60 & 3,44 & 8,39 & 3,30 & 8,49 & 2,85 \\
\hline \multirow[t]{2}{*}{$B^{\prime}$} & 5,74 & 2,82 & 6,60 & 3.29 & 7,72 & 3,47 & 8.57 & 3,35 & 8,86 & 2,94 \\
\hline & \multicolumn{10}{|c|}{$\mathrm{q}^{\prime}=100 \mathrm{kPa} \mathrm{b}=3,00 \mathrm{~m}$} \\
\hline B & 6,07 & 2,71 & 7.03 & 3,16 & 8,25 & 3,38 & 9,20 & 3,36 & 9,52 & 3,01 \\
\hline$B^{\prime}$ & 6,76 & 2,48 & 7,78 & 2,78 & 9,07 & 2,97 & 10,12 & 3,06 & 10,65 & 2,92 \\
\hline
\end{tabular}


d'ancrage fictif est plus grande que celle sur la surface de glissement intérieure inclinée. Cette différence devient encore plus claire avec l'introduction de surcharges plus élevées.

Enfin, on a pu démontrer que pour des surcharges élevées le modèle cinématique est de loin plus précis et plus économique que le modèle simplifié. La consistance de la cinématique des solides rigides est confirmée.

\section{3}

\section{Conclusion}

En se basant sur les résultats obtenus dans le cadre de ce travail, les recommandations suivantes sont proposées pour la vérification de la stabilité le long de la surface de glissement profonde :

- Les mécanismes de rupture doivent être analysés avec la méthode cinématique des solides rigides quí donne des résultats réalistes. Une modélisation correcte des mécanismes de rupture est assurée uniquement avec la méthode cinématique, comparée à la méthode simplifiée, parce qứelle permet d'un côté une utilisation correcte des résistances (résistance au cisaillement du sol dans les surfaces de glissement et les forces d'ancrages) et d'un autre côté elle est applicable aux différents problèmes de stabilité.

- La surface de glissement intérieure inclinée peut être remplacée par un mur d'ancrage vertical fictif si le solide actif situé à l'arrière n'est soumis à aucune charge et/ou aucune force d'ancrage (cas 1). Dans ce cas, le modèle simplifié est une bonne approche pour le modèle cinématique.

- Dans le cas où le mur est sollicité par des surcharges élevées (p. ex. $q^{\prime}=100 \mathrm{kPa}$ ), une modélisation correcte de la rupture est garantie seulement avec le modèle cinématique.

- Si le mur est sollicité par des surcharges movennes (p. ex. $q^{\prime}=30 \mathrm{kPa}$ ), on peut également étudier le modèle simplifié tant que le solide actif situé à l'arrière n'est soumis à aucune force d'ancrage.

Enfin, les études ont montré que l'utilisation du concept statistico-probabiliste de sécurité dans le dimensionnement et la vérification de stabilité des structures est de grande importance. Il permet en comparaison du concept conventionnel de sécurité, d'un côté une étude rationnelle et claire de la stabilité des structures et d'un autre côté une comparaison objective des différents modes de rupture d'une structure et même des différentes structures à travers un niveau de sécurité invariable et homogène.

\section{Bibliographie}

[1] Anderson W.F., Hanna T.H., AbdetMalek M.N. - a Overall stability of anchored retaining walls $\#$, Journal of geotechnical engineering [1], 1983, p. 1416-1433.

[2] Belabed L. - « Analyse de la fiabilité du système porteur "murs de soutènement à ancrage multiple levec les méthodes de probabilité n. Thèse de doctorat, Bauhaus-Universităt Weimar (Germany), 1996.

[3] Belabed L. - « Modélisation exacte des mécanismes de rupture pour les murs de soutènement à ancrage multiple 1. Bautechnik 73, 1996, p. 776-780.

[4] Belabed L. - « Application du concept probabiliste de sécurité sur la vérification de la stabilité d'ensemble des murs de soutènement ancrés avec la cinématique des solides rigides 3. Revue française de Géotechnique n 89 , 1999, p. 4954
[5] CEN - « Geotechnical design, general rules. European Committee for Standardization (CEN) ». Eurocode. Nederiands Normalisatie-instituut (NNI), Delft, Prestandard (ENV), 1994.

[6] DIN 1054.100 - "Vérifications de la sécurité en mécanique des sols $n$. Institut allemand de normalisation (DIN), 1995.

7] Gäbler G - « Murs cloutés. Comporte. ment et stabilité s. Publications de l'Ins. titut de mécanique des sols et des roches, Université de Kar!sruhe, n $108,1987$.

[8] Gäbler G., Gudehus G. - " Anchored Walls. Model tests and statistica! design 》. Proceedings of the 12 th International Conference on Soil Mechanics and Foundation Engineering, Rio de Janeiro, 1989, p. 829-832.

[9] Goldscheider M. Kolymbas D. - "Calcul de la stabilité des murs de soutènement à ancrage multiple n. Geotechnik 4 . 1980, p. $156-164$.
[10] Grusibau - Règles fixant les exigences en matière de sécurité des ouvrages de construction. Institut allemand de normalisation (DIN) e.V. Beuth Verlag, Berlin-Köln, 1981.

[11] Gudehus G. - Détermination de la pression des terres. Manuel des fondations, tome 1, version 4, Ernst \& Sohn, Berlin, 1990, p. 289-361.

[12] Habib P. (Ed.) - Recommendations for the design, calculation, construction and monitoring of ground anchorages (trans. lation from French). Rotterdam, Brooklield, A.A. Balkema, 1989.

[13] Kranz E. - u. Sur l'ancrage des rideaux de palplanches 3), Informations constructions hycirauliques et recherches, $n^{3} 11$. version 2, Berlin, Ernst \& Sohn, 1953.

[14] Weîss W. - « Evvaluation de la sécurité pour les fondations 3. Publications de Institut des fondations de l'université Technique de Berlin, $n^{\circ}$ 20, 1991. 